\title{
Correlation of Ultrasound with MRI for the Evaluation of Meniscal Tears
}

\author{
Suresh A ${ }^{1}$, Aishwarya Shetty ${ }^{2}$, Prachi Kala ${ }^{3}$ \\ ${ }^{1}$ Associate professor, ${ }^{2}$ Post Graduate, ${ }^{3}$ Professor and Head of Department, Radiology department, Vydehi institute of medical sciences and Research centre, \\ Bangalore, India.
}

\section{Abstract}

Background: Objective: To see the diagnostic value along with the sensitivity and specificity values of ultrasound in comparison to MRI for the evaluation of meniscal tears. Subjects and Methods: The study examined 70 patients who presented with history of trauma and suspected injury to the menisci. Patients were examined initially with ultrasound followed by non-contrast magnetic resonance imaging (MRI), with MRI used as a reference standard. Results: A total of 52 medial meniscal tears were detected on MRI out of which 42 cases were detected correctly on ultrasound giving a specificity of $80.8 \%$; likewise, a total of 51 patients were detected with lateral meniscal tears on MRI out of which 38 cases were detected correctly on ultrasound giving a specificity of $74.5 \%$. Conclusion: Ultrasound is a reliable imaging modality in identifying medial and lateral meniscal tears.

Keywords: Meniscal Tear, MRI, Ultrasound..

Corresponding Author: Dr. Aishwarya Shetty, Post Graduate, Radiology department, Vydehi institute of medical sciences and Research centre, Bangalore, India.

Received: September 2018

Accepted: December 2018

\section{Introduction}

The knee joint is a complex synovial hinge joint with its key role being to maintain stability during various weight bearing circumstances and is one of the most common sites that are affected in post-traumatic injuries by athletic and non-athletic individuals. It is important to be familiar with the anatomical components that comprise of the knee joint to understand the mechanism behind post traumatic injuries. The menisci are fibrocartilaginous structures on the medial and lateral aspect within the knee joint which help in load transmission and assisting in stabilizing the knee joint. ${ }^{[1]}$

The medial meniscus gives a C-shaped configuration and lateral menisci with an O-shaped configuration; both consisting of an anterior and posterior horn with the posterior horn typically being larger than the anterior horn. ${ }^{[2]}$

Prompt diagnosis is important in the evaluation of meniscal tears in post-traumatic injuries to prevent further complications and decide whether surgical intervention is indicated.

MRI is the imaging modality of choice for the evaluation and grading of meniscal tears however, limitations associated with MRI include metallic implants, motion artifacts, claustrophobia and is expensive in comparison to ultrasound. Ultrasound has become a reliable non-invasive source in the diagnosis of meniscal injuries with many advantages of its own mainly being its wide availability, low cost and the use of real-time imaging. ${ }^{[3]}$

\section{Subjects and Methods}

Across sectional study was conducted on 70 patients (60 males and 10 females; aged from 14-72 years) with clinically suspected meniscal tears referred to the department of radiodiagnosis in Vydehi institute of medical sciences and research Centre (VIMS \& RC).

The exclusion criteria were patients diagnosed with fractures on plain radiograph, degenerative or inflammatory changes on plain radiograph, patients with cardiac implants and metallic implants and post-operative patients.

Ultrasound was initially performed on the patients with Philips HD15 ultrasound machine using a superficial 3$12 \mathrm{MHz}$ linear transducer.

To check the medial and lateral menisci the patient was put in sitting position with the knees flexed at 90 degrees and the medial and lateral compartments were evaluated.

The criteria to for the diagnosis of meniscal tears included irregular/ hazy contour and presence of a meniscal cyst.

This was followed by an MRI on the same day with a Philips Achieva 1.5 Tesla MRI scanner.

The patient was placed supine with 10-15 degrees flexion of the knee with the lower border of the patella being the centre of the beam localizer. On MRI the diagnosis of meniscal tears was based on hyperintense signal changes which may be longitudinal, horizontal or radial in shape; with or without extending to the articular surface depending on the severity of the meniscal tear. Displaced tears give a bucket handle, flap or parrot beak configuration. 


\section{Suresh et al; Carrelation of Ultrasound with MRI for the Evaluation of Meniscal Tears}

The results of ultrasound and MRI were compared with the following diagnostic indices: sensitivity, specificity, positive predictive value and negative predictive values.

\section{Results}

Table 1: Comparison between USG and MRI in diagnosis of Normal Medial Meniscus

\begin{tabular}{|c|c|c|c|c|c|}
\hline \multicolumn{2}{|c|}{} & \multicolumn{4}{|c|}{ MRI } \\
\cline { 3 - 6 } \multicolumn{2}{|c|}{} & \multicolumn{2}{|c|}{ Present } & \multicolumn{2}{c|}{ Absent } \\
\cline { 3 - 6 } & Count & $\mathbf{\%}$ & Count & $\mathbf{\%}$ \\
\hline \multirow{2}{*}{ Ultrasound } & Present & 18 & $100.0 \%$ & 10 & $19.2 \%$ \\
\cline { 2 - 6 } & Absent & 0 & $0.0 \%$ & 42 & $80.8 \%$ \\
\hline
\end{tabular}

[Table 1] shows that out of 18 patients that were normal for medial meniscal tears on MRI, $18(100 \%)$ were normal on ultrasound as well giving a sensitivity of $100 \%$. Out of the 52 patient that showed abnormal findings on ultrasound 42 were diagnosed correctly on ultrasound giving a specificity of $80.8 \%$. Positive predictive value: $64.3 \%$, Negative predictive value: $100 \%$.

Table 2: Comparison between USG and MRI in diagnosis of Partial Tear of Medial Meniscus

\begin{tabular}{|c|c|c|c|c|c|}
\hline \multicolumn{2}{|c|}{} & \multicolumn{4}{|c|}{ MRI } \\
\cline { 3 - 6 } \multicolumn{2}{|c|}{} & \multicolumn{2}{|c|}{ Present } & \multicolumn{2}{c|}{ Absent } \\
\cline { 3 - 6 } \multicolumn{2}{|c|}{} & Count & $\mathbf{\%}$ & Count & $\mathbf{\%}$ \\
\hline \multirow{2}{*}{ Ultrasound } & Present & 25 & $78.1 \%$ & 0 & $0.0 \%$ \\
\cline { 2 - 6 } & Absent & 7 & $21.9 \%$ & 38 & $100.0 \%$ \\
\hline
\end{tabular}

[Table 2] shows that out of 32 subjects with partial tear for medial meniscus on MRI, $78.1 \%$ (sensitivity) were positive on USG and $21.9 \%$ were negative on USG. Out of 38 subjects without partial tear in Medial meniscus, 100\% (specificity) were negative for partial tear in USG. Positive predictive value: $100 \%$, Negative predictive value: $84.4 \%$.

Table 3: Comparison between USG and MRI in diagnosis of Complete Tear of Medial Meniscus.

\begin{tabular}{|c|c|c|c|c|c|}
\hline \multirow{2}{*}{} & \multicolumn{4}{|c|}{ MRI } \\
\cline { 3 - 6 } \multicolumn{2}{|c|}{} & \multicolumn{2}{|c|}{ Present } & \multicolumn{2}{c|}{ Absent } \\
\cline { 3 - 6 } & Count & $\mathbf{\%}$ & Count & \% \\
\hline \multirow{2}{*}{ Ultrasound } & Present & 19 & $95.0 \%$ & 0 & $0.0 \%$ \\
\cline { 2 - 6 } & Absent & 1 & $5.0 \%$ & 50 & $100.0 \%$ \\
\hline
\end{tabular}

[Table 3] shows out of 20 subjects with complete tear of medial meniscus on MRI, 95\% (sensitivity) were positive in USG and $5 \%$ were negative on USG. Out of 50 subjects without complete tear of Medial meniscus, 100\% (specificity) were negative for complete tear on USG. Positive predictive value: $100 \%$, Negative predictive value: $98 \%$.

Table 4: Comparison between USG and MRI in diagnosis of Normal Lateral Meniscus

\begin{tabular}{|c|c|c|c|c|c|}
\hline \multicolumn{2}{|c|}{} & \multicolumn{3}{|c|}{ MRI } \\
\cline { 3 - 6 } \multicolumn{2}{|c|}{} & \multicolumn{2}{|c|}{ Present } & \multicolumn{2}{c|}{ bsent } \\
\cline { 3 - 6 } \multicolumn{2}{|c|}{ Ultrasound } & Count & \% & Count & \% \\
\cline { 2 - 6 } & Absent & 18 & $94.7 \%$ & 13 & $25.5 \%$ \\
\hline
\end{tabular}

Out of 19 subjects with normal lateral meniscus in MRI, $94.7 \%$ (sensitivity) were positive in USG and 5.3\% were negative in USG. Out of 51 subjects without Normal Lateral meniscus, $74.5 \%$ (specificity) were negative and $25.5 \%$ were positive for pathology in USG. Positive predictive value: $100 \%$, Negative predictive value: $98 \%$.

Table 5: Comparison between USG and MRI in diagnosis of Partial Tear of Lateral Meniscus

\begin{tabular}{|c|c|c|c|c|c|}
\hline \multicolumn{2}{|c|}{} & \multicolumn{4}{|c|}{ MRI } \\
\cline { 3 - 6 } \multicolumn{2}{|c|}{} & \multicolumn{2}{|c|}{ Present } & \multicolumn{2}{c|}{ Absent } \\
\cline { 3 - 6 } & Count & $\mathbf{\%}$ & Count & \% \\
\hline \multirow{2}{*}{ Ultrasound } & Present & 22 & $62.9 \%$ & 1 & $2.9 \%$ \\
\cline { 3 - 6 } & Absent & 13 & $37.1 \%$ & 34 & $97.1 \%$ \\
\hline
\end{tabular}

Out of 35 subjects with partial tear lateral meniscus in MRI, $62.9 \%$ (sensitivity) were positive on USG and $37.1 \%$ were negative on USG. Out of 35 subjects without partial tear in lateral meniscus, $97.1 \%$ (specificity) were negative for partial tear and $2.9 \%$ were positive for partial tear in USG. Positive predictive value: $95.6 \%$, Negative predictive value: $72.3 \%$.

Table 6: Comparison between USG and MRI in diagnosis of Complete Tear of Lateral Meniscus

\begin{tabular}{|c|c|c|c|c|c|}
\hline \multicolumn{2}{|c|}{} & \multicolumn{4}{|c|}{ MRI } \\
\cline { 3 - 6 } \multicolumn{2}{|c|}{} & \multicolumn{2}{|c|}{ Present } & \multicolumn{2}{c|}{ Absent } \\
\cline { 3 - 6 } \multicolumn{2}{|c|}{} & Count & \% & Count & \% \\
\hline \multirow{2}{*}{ Ultrasound } & Present & 14 & $100.0 \%$ & 0 & $0.0 \%$ \\
\cline { 2 - 6 } & Absent & 2 & $0.0 \%$ & 54 & $100.0 \%$ \\
\hline
\end{tabular}

Out of 14 subjects with complete tear of lateral meniscus on MRI, $87.5 \%$ (sensitivity) were positive on USG and 12.5\% were negative on USG. Out of 54 subjects without Full tear in lateral meniscus, $100 \%$ (specificity) were negative for Full tear on USG. Positive predictive value: 100\%, Negative predictive value: $96.4 \%$.

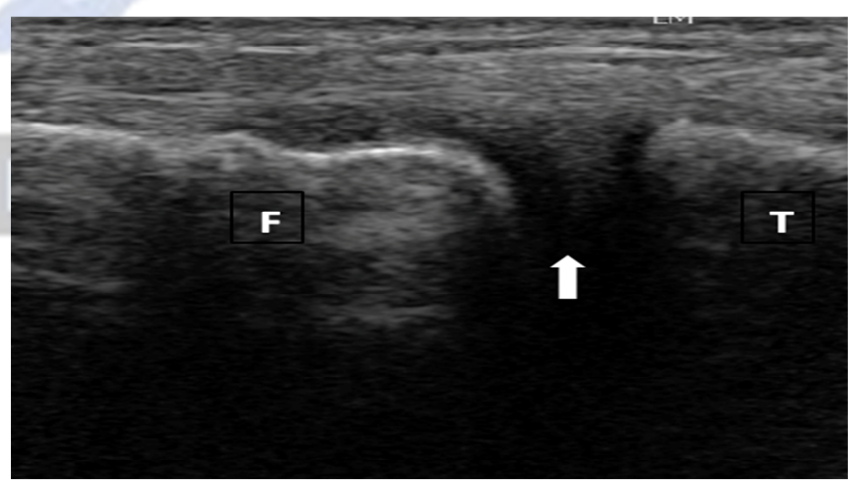

Figure 1: Ultrasound image with superficial probe showing normal contour of lateral meniscus (arrow). (Source - VIMS \& RC)

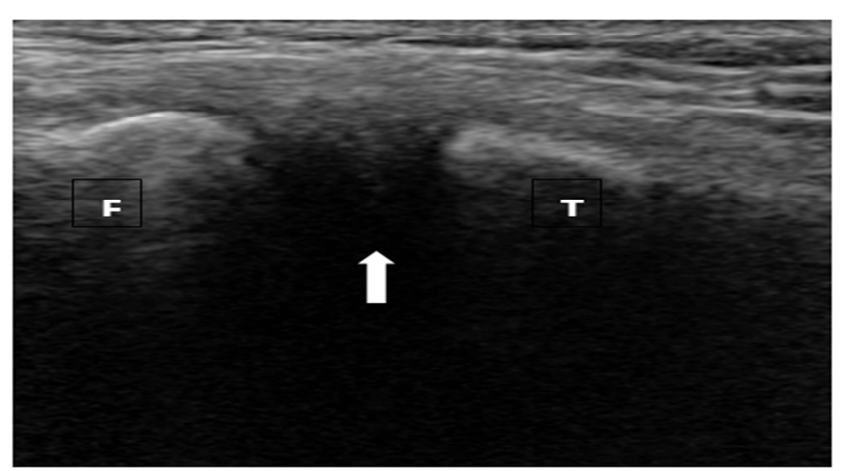

Figure 2: Ultrasound, superficial probe: tear of lateral meniscus showing irregular contour (arrow). (Source - VIMS \& RC) 


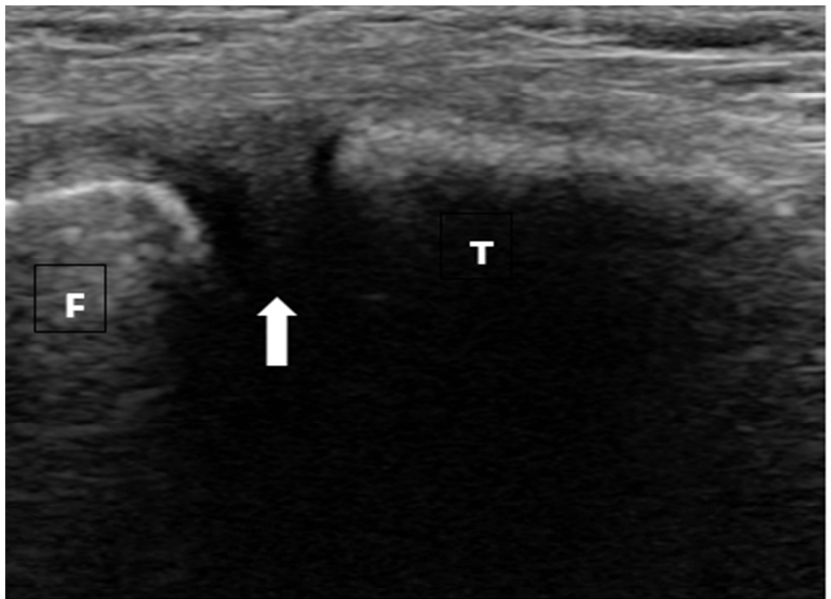

Figure 3: Ultrasound image with superficial probe showing normal contour of medial meniscus (arrow). (Source - VIMS \& RC)

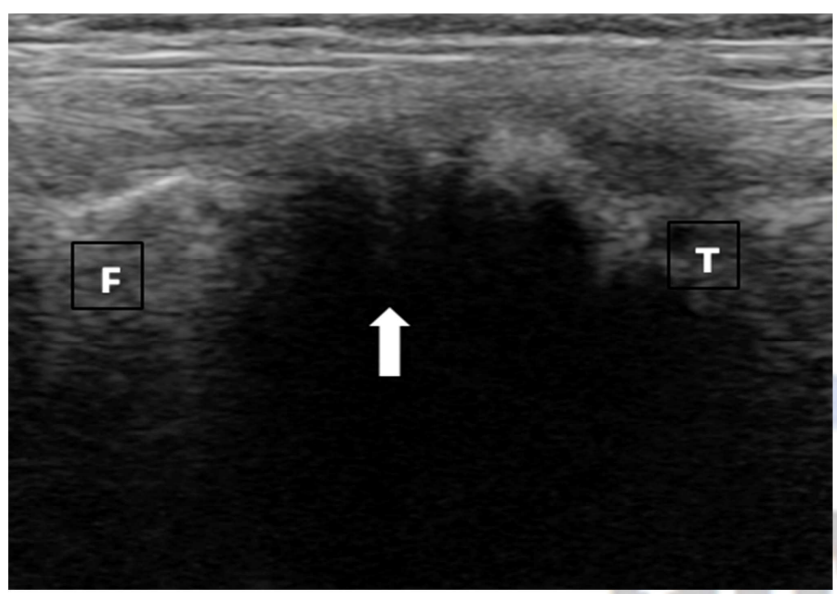

Figure 4: Ultrasound, superficial probe: tear of medial meniscus showing irregular contour (arrow). (Source - VIMS \& RC)

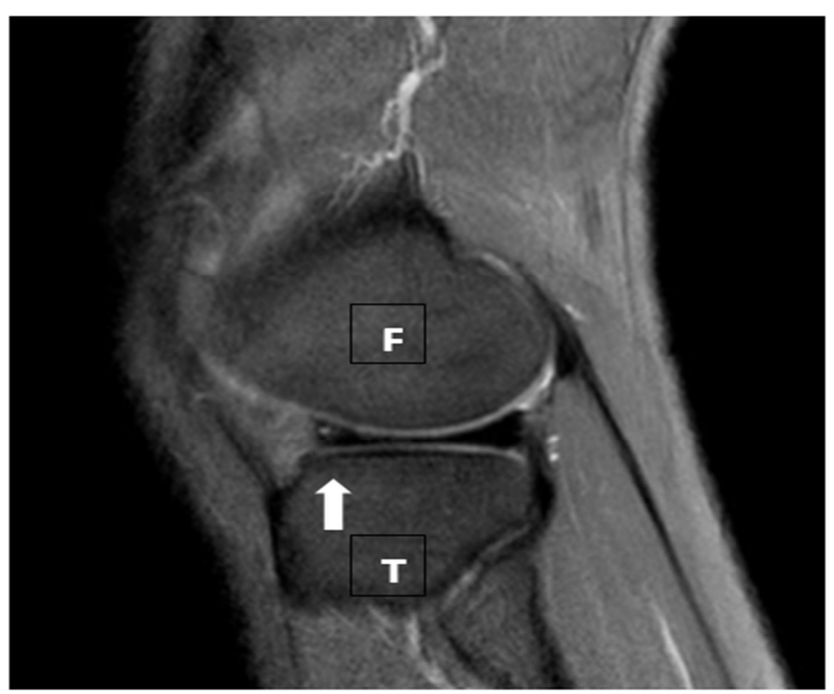

Figure 5: Sagittal PDW_mSPIR MR showing Grade I horizontal tear involving anterior horn of lateral meniscus (arrow). (Source - VIMS \& RC)

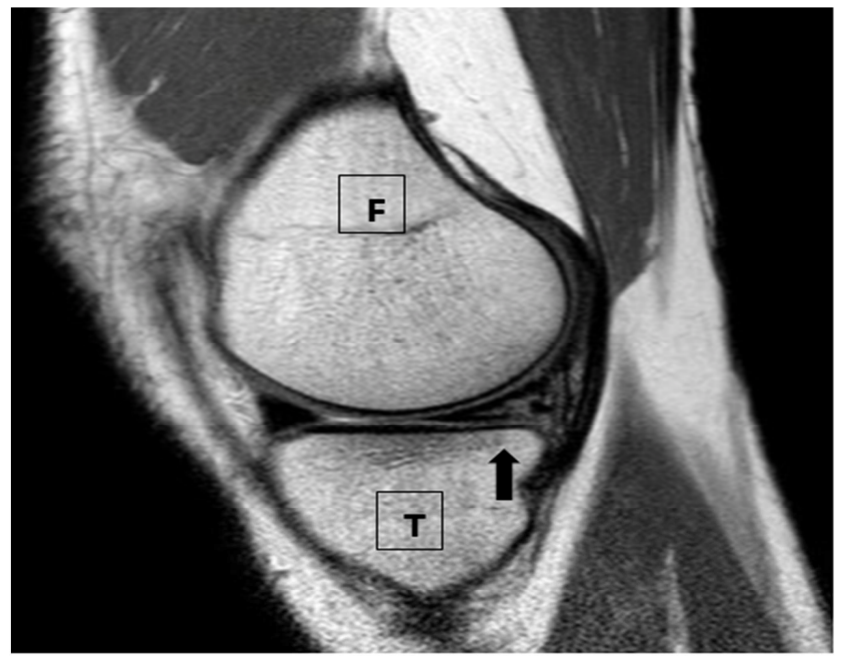

Figure 6: Sagittal PDW aTSE MR showing Grade IIc horizontal tear of the posterior horn of medial meniscus (arrow). (Source - VIMS \& RC)

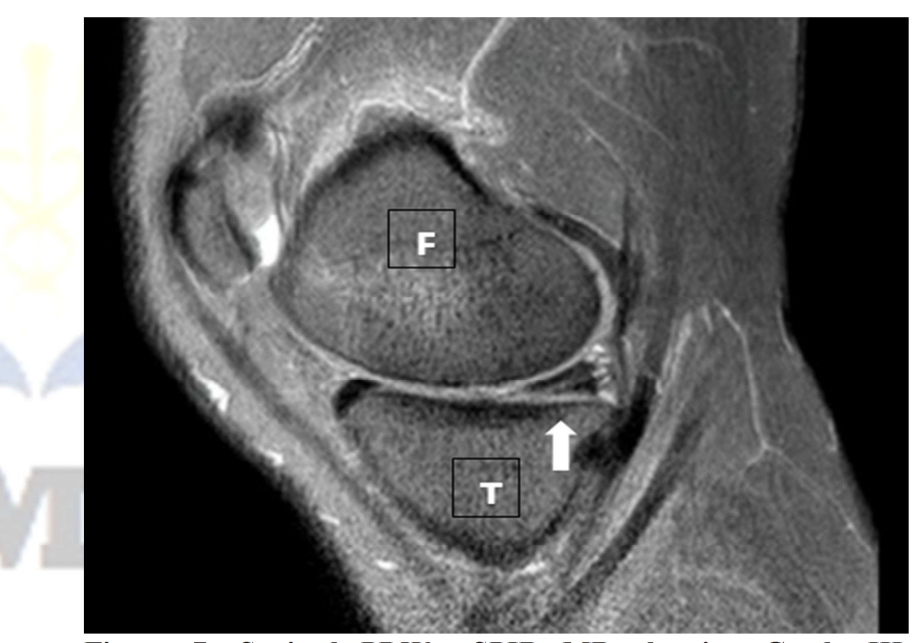

Figure 7: Sagittal PDW_mSPIR MR showing Grade III horizontal tear of posterior horn of medial meniscus (arrow). (Source - VIMS \& RC)

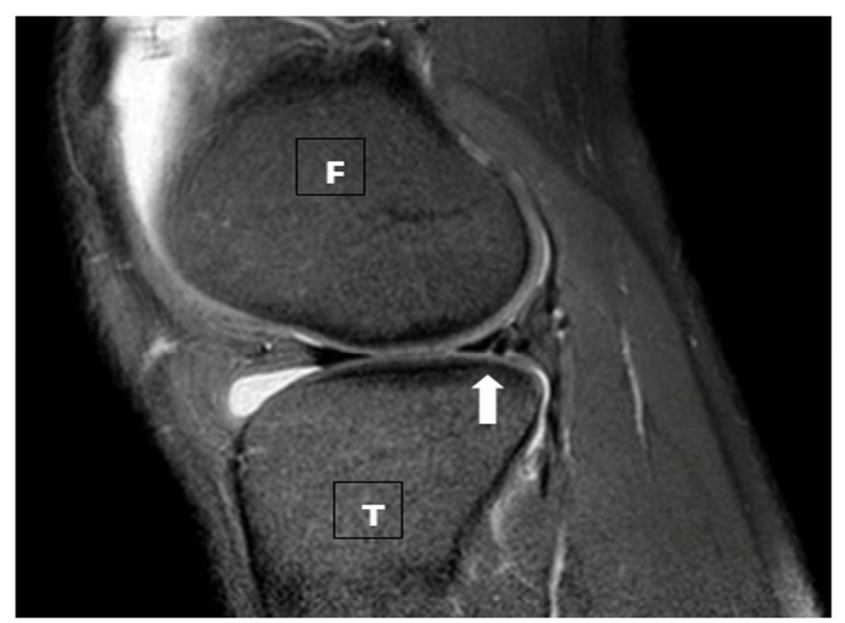

Figure 8: Sagittal PDW_mSPIR MR showing vertical tear of posterior horn of lateral meniscus (arrow). (Source - VIMS \& RC) 


\section{Discussion}

Ultrasound has been used in musculoskeletal imaging and with its wide availability and low cost continues to be a common method for evaluation.

From the results it is evident that ultrasound is an effective method in evaluation of meniscal tears with high sensitivity and specificity values in comparison to MRI which has been set as the Gold standard in this study.

Other studies with similar findings have been done to evaluate ultrasound in the diagnosis of injury to the knee joint and its various components including the ligaments, cartilage and menisci, for example Ahmed Alizadeh et al conducted a study on 74 patients for the diagnosis of medial meniscal tears; the ultrasound and MRI findings were compared with arthroscopic results. The study showed high sensitivity and specificity values for ultrasound of $100 \%$ and $88.8 \%$ respectively in the age category above 30 years; $83.3 \%$ and $71.4 \%$ respectively in the age category below 30 years, concluding that ultrasound is a reliable method in suspected cases of medial meniscal injuries. ${ }^{[4]}$

Another study by Park G-Y et al where ultrasound was performed on 22 patients and compared with MRI for the evaluation of meniscal tears.

Results showed 29 meniscal tears on MRI out of which 25 were correctly diagnosed on ultrasound showing high sensitivity and specificity values of $86.2 \%$ and $84.9 \%$ respectively deducing that ultrasound is an effective imaging modality in diagnosing meniscal tears. ${ }^{[5]}$

The study showed the greater the degree of meniscal tear the more evident the injury was on ultrasound therefore; complete meniscal tears were more easily detected on ultrasound as compared to partial tears. This showed that the accuracy of ultrasound was higher with increased severity of meniscal injuries.

The accuracy of ultrasound to detect normal cases was higher than the ability of ultrasound to detect abnormal cases which is evident by the higher specificity values for partial and complete meniscal tears as compared to the sensitivity values.

In our study the ultrasound accuracy based on the sensitivity, specificity, positive predictive value and negative predictive values gave a p-value of $<0.05$; which represents a significant association between ultrasound and MRI in the evaluation of meniscal tears.

\section{Conclusion}

Ultrasound is an effective imaging modality in the evaluation of meniscal tears in post-traumatic patients with many advantages of its own in comparison to MRI.

\section{References}

1. Abdulhasan JF, Grey MJ. Anatomy and physiology of knee stability. Journal of Functional Morphology and Kinesiology 2017;2,34:1-11.

2. Razek A.A.K.A, Fouda NS, Elmetwaley N, Elbogdady E. Sonography of the knee joint. Journal of ultrasound 2009; 12: 53-60.

3. Nazarian LN. The top 10 reasons musculoskeletal sonography is an important complementary or alternative technique to MRI. AJR 2008; 190:1622-1625

4. Alizadeh A, Jandaghi AB, Zirak AK, Karimi A, Mardani-Kivi M, Rajabzadeh A. Knee sonography as a diagnostic test for medial meniscal tears in young patients. Eur J Orthop Surg Traumatol 2013;23(8):92731.

5. Park GY, Kim JM, Lee SM, Lee MY. The value of ultrasonography in the detection of meniscal tears diagnosed by magnetic resonance imaging. Am J Phys Med Rehabil 2008;87:14-20.

Copyright: () the author(s), publisher. Asian Journal of Medical Radiological Research is an Official Publication of "Society for Health Care \& Research Development". It is an open-access article distributed under the terms of the Creative Commons Attribution Non-Commercial License, which permits unrestricted non-commercial use, distribution, and reproduction in any medium, provided the original work is properly cited.

How to cite this article: Suresh A, Shetty A, Kala P. Correlation of Ultrasound with MRI for the Evaluation of Meniscal Tears. Asian J. Med. Radiol. Res. 2018;6(2):23-26.

DOI: dx.doi.org/10.21276/ajmrr.2018.6.2.7

Source of Support: Nil, Conflict of Interest: None declared. 